\title{
LINFADENECTOMIA NO ADENOCARCINOMA GÁSTRICO
}

\author{
Elias Jirjoss lluas*, Carlos Alberto Malheiros, Paulo Kassab, Osvaldo Antonio Prado Castro
}

Estudo realizado no departamento de Cirurgia da Faculdade de Ciências Médicas da Santa Casa de São Paulo, São Paulo, SP

*Correspondência:

Rua Passo da Pátria, 1432, apto. $81-\mathrm{Cl}$

Cep 05085-000 - São Paulo - SP

eliasjilias@yahoo.com.br

\begin{abstract}
RESUMO
Овرetivos. Realizar uma simulação de linfadenectomia DI em 57 pacientes submetidos a gastrectomia com linfadenectomia D2 e analisar a migração do estádio na classificação da Associação Japonesa de Câncer Gástrico (JGCA).

Métodos. Os pacientes foram submetidos a linfadenectomia D2 entre agosto de 1997 e novembro de 2001 na Faculdade de Medicina da UNISA. Os linfonodos eram dissecados pelo próprio cirurgião, selecionados de acordo com a estação linfonodal. Analisaram-se as variáveis: sexo, idade, comprometimento linfonodal e número de linfonodos dissecados. Resultados. Após a simulação de linfadenectomia DI realizou-se novo estadiamento para estudar a migração na classificaçãa da JGCA. Os tumores precoces corresponderam a 19,3\% dos casos e os avançados a 80,7\%. Linfonodos positivos em NI e N2 em 43,9\% dos pacientes. A média de linfonodos dissecados foi de 28,63 por paciente. Nos tumores IA, IB e II não houve mudança no estádio após a simulação DI. Nos tumores IIIA, de dez pacientes, três migraram para estádios II e IB; nos IIIB, houve migração de 18 em 2l; e, no estádio IV, todos migraram para IIIB.

Conclusão. Linfadenectomia D2 é importante para o estadiamento mais apurado do câncer gástrico, principalmente nos tumores avançados. A simulaçãa de linfadenectomia DI alterou o estádio dos tumores avançados sendo que os estádios que mais sofreram migração na classificação da JGCA foram o IIIA, IIIB e IV. A linfadenectomia D2 evitou a presença de doença residual nos linfonodos após a cirurgia em quase metade dos pacientes.
\end{abstract}

UniteRmos: Excisão de linfonodo. Adenocarcinoma. Neoplasias gástricas. Gastrectomia.

\section{INTRODUÇÃO}

O câncer gástrico é um dos tumores malignos com maior incidência, principalmente na América Central e do Sul, Japão, Coréla e países bálticos'.

O Instituto Nacional de Câncer (INCA)2 estimou a incidência de neoplasia maligna do estômago no Estado de São Paulo para o ano de 2003 em 23,88 indivíduos por 100.000 homens e 8,91 a 12,29 por 100.000 mulheres.

Atualmente, nos Estados Unidos, a taxa de sobrevida de cinco anos após tratamento cirúrgico curativo do câncer de estômago gira em torno de $20 \%$ a 25\%. No Japão, o diagnóstico precoce e o tratamento cirúrgico agressivo, incluindo linfadenectomia, resultaram em uma taxa de sobrevida de cinco anos superior a $50 \%{ }^{3}$.

Os cirurgiões japoneses efetuaram grandes esforços para a melhora do tratamento cirúrgico do câncer gástrico. As taxas de sobrevida em cinco anos, que eram de 44\% entre 1963 e 1973, subiram para 56\% entre 1969 e 1973 e para $61 \%$ entre 197| e 1985'. Atualmente, a sobrevida em cinco anos, no Japão, apenas com o tratamento cirúrgico encontra-se em torno de $71 \%$.

Recidivas precoces nos linfonodos ocorreram em $30 \%$ dos casos, enquanto que as recidivas tardias ocorreram em $40 \%$ nesses locais ${ }^{6,7}$.

A técnica de dissecção linfonodal DI consiste na ressecção em bloco dos nódulos das cadeias NI (até $3 \mathrm{~cm}$ do tumor), que depende da localização do tumor primário. $O$ pâncreas e o baço devem ser preservados, a menos que haja invasão direta desses órgãos. A artéria gástrica esquerda deve ser ligada na sua bifurcação nos ramos ascendente e descendente. A dissecção D2 inclui a ressecção das cadeias linfonodais $\mathrm{NI}$ e $\mathrm{N} 2$ (até $6 \mathrm{~cm}$ do tumor primário), incluindo os linfonodos ao longo das artérias gástrica esquerda, hepática comum e esplênica e os ao redor do tronco celíaco ${ }^{8}$.

Msika e Kianmanesh ${ }^{9}$ analisaram diversos estudos e concluíram que a linfadenectomia D2 leva a um bom estadiamento da neoplasia gástrica.

Diversos estudos mostraram que os maus resultados nas linfadenectomias D2 foram influenciados pela retirada do baço e do pâncreas durante a cirurgia, além da pouca experiência desses centros na realização de linfadenectomias regradas $10,11,12,13,14$.

Parece haver um aumento da sobrevida nos pacientes submetidos a gastrectomia com linfadenectomia D2, principalmente quando o tumor invade a camada muscular do estômago e a serosa sem comprometer estruturas vizinhas ou quando atinge menos de 15 linfonodos ${ }^{15}$.

A União Internacional de Combate ao Câncer (UICC) tem substituído os sítios linfonodais comprometidos pelo número de linfonodos comprometidos como melhor indicador prognóstico no câncer de estômago ${ }^{16}$.

A linfadenectomia trouxe uma grande contribuição no prognóstico, particularmente nos pacientes com câncer de estômago nos estádios T2 e T3 ${ }^{17}$.

Estudo realizado em 1038 pacientes operados no período de 1985 a 1999, com ressecção R0 mostrou que o número de linfonodos comprometidos é o que melhor define o prognóstico. As estimativas de sobrevida baseadas no número de linfonodos comprometidos foram melhor representadas quando ao menos 15 linfonodos foram examinados ${ }^{18}$.

Degiuli et al. ${ }^{14}$ fizeram estudo multicêntrico randomizado em que compararam a morbidade e a mortalidade em dois grupos de pacientes: um submetido a gastrectomia com linfadenectomia DI e outro a 


\begin{tabular}{|c|c|c|c|c|c|c|c|c|c|c|}
\hline \multicolumn{11}{|c|}{$\begin{array}{c}\text { Tabela I - Estadiamento segundo a JGCA dos } 57 \text { pacientes com adenocarcinoma gástrico submetidos a linfadenectomia D2 } \\
\text { e novo estadiamento após simulação de linfadenectomia DI. FM-UNISA, 2003. }\end{array}$} \\
\hline \multicolumn{3}{|c|}{$\begin{array}{l}\text { Comportamento } \\
\text { linfonodal }\end{array}$} & \multirow[t]{2}{*}{$\begin{array}{l}\text { Classificação pela JGCA } \\
\text { após linfadenectomia D2 }\end{array}$} & \multicolumn{6}{|c|}{$\begin{array}{c}\text { Classificação pela JGCA } \\
\text { com simulação de linfadenectomia DI }\end{array}$} & \multirow[b]{2}{*}{ Tota } \\
\hline & $\mathrm{NI}$ & N2 & & la & Ib & II & IIla & Illb & IV & \\
\hline $\mathbf{T}$ & & & & & & & & & & \\
\hline \multirow[t]{2}{*}{ i } & $(-)$ & $(-)$ & la & 11 & & & & & & 11 \\
\hline & $(-)$ & $(-)$ & $\mathrm{Ib}$ & & 5 & & & & & 5 \\
\hline $\mathbf{T}$ & $(-)$ & $(+)$ & \|\|$_{a}$ & & |* & & & & & 1 \\
\hline \multirow[t]{3}{*}{2} & $(t)$ & $(t)$ & IIla & & & $2 *$ & & & & 2 \\
\hline & $(+)$ & $(-)$ & $\|$ & & & 2 & & & & 2 \\
\hline & $(-)$ & $(-)$ & $\|$ & & & 4 & & & & 4 \\
\hline $\mathbf{T}$ & $(t)$ & $(t)$ & $\|l\| b$ & & & & $18 *$ & 1 & & 19 \\
\hline 3 & $(t)$ & $(-)$ & $\| l l a$ & & & & 7 & & & 7 \\
\hline$T$ & $(+)$ & $(+)$ & IV & & & & & 4* & & 4 \\
\hline 4 & $(+)$ & $(-)$ & $\| l l b$ & & & & & 2 & & 2 \\
\hline Total & & & & 11 & 6 & 8 & 25 & 7 & 0 & 57 \\
\hline
\end{tabular}

(-) negativo para neoplasia; ( + ) positivo para neoplasia

T: profundidade do tumor na parede gástrica

* Pacientes em que houve mudança no estadiamento após a simulação da linfadenectomia DI

gastrectomia com linfadenectomia D2. As complicações foram pequenas e não houve diferença estatisticamente significante entre os dois grupos quanto à morbi-mortalidade. Os autores concluíram que, em centros experientes, a morbi-mortalidade da gastrectomia com linfadenectomia D2 não é maior que a DI e comparável aos resultados japoneses.

A ressecção cirúrgica do câncer de estômago com linfadenectomia D2 realizada por cirurgião experiente mostrou baixa morbidade e maior sobrevida em pacientes portadores de neoplasia gástrica ${ }^{19}$.

\section{Objetivo}

Realizar simulação de linfadenectomia DI em 57 pacientes submetidos a gastrectomia com linfadenectomia D2 por câncer de estômago e analisar a migração do estádio na classificação da Associação Japonesa de Câncer Gástrico (JGCA) após essa simulação. Também estudou-se a incidência de doença residual em linfonodos caso não se realizasse a linfadenectomia D2.

\section{Métodos}

Foram estudados prospectivamente 57 pacientes com adenocarcinoma gástrico operados no período de agosto de 1997 a novembro de 2001 pela Disciplina de Cirurgia Geral da Faculdade de Medicina da Universidade Santo Amaro. Dos 57 pacientes, 50,9\% eram do sexo masculino e 49, $1 \%$ do sexo feminino. A média de idade foi de 58,3 anos (variando de 31 a 84 anos).

A cirurgia era realizada por meio de incisão mediana, supra e infra umbilical (do processo xifóide até cerca de $3 \mathrm{~cm}$ abaixo da cicatriz umbilical). O primeiro passo após a laparotomia consistiu em realizar lavado peritoneal supra e infra-umbilical com 100 a $200 \mathrm{ml}$ de solução salina a $0,9 \%$. Todos os pacientes foram submetidos a gastrectomia subtotal ou total com linfadenectomia D2. A escolha do procedimento cirúrgico obedeceu os princípios determinados pela Associação Japo- nesa de Câncer Gástrico (JGCA). A determinação dos grupos linfonodais a serem dissecados era estabelecida após certificação exata da localização do tumor no estômago. Todos os pacientes foram submetidos à drenagem da cavidade abdominal.

Imediatamente após o ato operatório, o cirurgião dissecava minuciosamente a peça cirúrgica retirando todas as cadeias linfonodais, individualizando cada uma delas de acordo com o protocolo do Centro Nacional do Câncer de Tóquio, Japão.

Os linfonodos de cada estação foram dissecados, colocados em frascos com formol a 4\% separados de acordo com sua localização, identificados e encaminhados à análise anatomopatológica para se averiguar a presença de metástase tumoral. Todas as peças cirúrgicas foram analisadas por patologistas com treinamento em câncer de estômago.

Analisou-se o comportamento linfonodal após a linfadenectomia D2 e o número de linfonodos extirpados.

Foram estudadas, também, as características do comprometimento linfonodal de cada grupo de pacientes de acordo com a profundidade do tumor (T). Nestes grupos, também foi feito estadiamento segundo a JGCA, como recomendado pela Associação Brasileira de Câncer Gástrico em 2002.

Após o estadiamento, foi realizada nestes pacientes uma simulação de linfadenectomia DI e foi estudada a mudança de estadiamento nos pacientes como um todo e em cada grupo $T$ em particular.

\section{Resultados}

Com relação à profundidade dos tumores, 19,3\% acometiam a camada mucosa ( $\mathrm{TI}$ ), 17,5\% a camada muscular (T2), 52,6\% a serosa (T3) e 10,5\% eram T4. Vinte pacientes não apresentaram comprometimento linfonodal, 19,3\% apresentaram comprometimento no nível I e 43,9\% apresentaram comprometimento linfonodal nos níveis I e 2. Um paciente 


\section{Tabela 2 - Análise de concordância entre a classificação pela JGCA após linfadenectomia D2 e a classificação pela simulação DI dos 57 pacientes com adenocarcinoma gástrico. FM-UNISA, 2003}

\begin{tabular}{|c|c|c|c|c|c|c|}
\hline \multirow[t]{2}{*}{$\begin{array}{l}\text { Classificação pela JGCA } \\
\text { após linfadenectomia D2 }\end{array}$} & \multicolumn{5}{|c|}{$\begin{array}{c}\text { Classificação pela JGCA com } \\
\text { simulação de linfadenectomia DI }\end{array}$} & \multirow[b]{2}{*}{ Total } \\
\hline & la & Ib & II & IIla & Illb & \\
\hline $\begin{array}{l}\text { la } \\
\mathrm{lb}\end{array}$ & 11 & 5 & & & & $\begin{array}{l}11 \\
5\end{array}$ \\
\hline II & & & 6 & & & 6 \\
\hline Illa & & |* & 2* & 7 & & 10 \\
\hline Illb & & & & $18 *$ & 3 & 21 \\
\hline IV & & & & & 4* & 4 \\
\hline Total & 11 & 6 & 8 & 25 & 7 & 57 \\
\hline
\end{tabular}

( I,8\%) apresentou comprometimento no nível 2, porém não nos níveis I . Foram dissecados uma média de 28,63 linfonodos por caso ( $D P=14,79)$.

As Tabelas I e 2 mostram as alterações que ocorreram nos estádios após a simulação da linfadenectomia DI nos pacientes que haviam sido submetidos a gastrectomia com linfadenectomia D2.

\section{Discussão}

A cirurgia é o único procedimento isolado que pode levar à cura ou a uma maior sobrevida. O prognóstico após uma ressecção completa do tumor macro e microscopicamente ( $\mathrm{R}$ 0) está diretamente relacionado à penetração da serosa (T3) e ao envolvimento linfonodal. A sobrevida de cinco anos após a cirurgia varia de mais de $90 \%$ em câncer precoce até menos de $20 \%$ em pacientes com câncer avançado. Em vista deste pobre prognóstico em doentes portadores de câncer avançado, outros tipos de tratamento têm sido considerados para indivíduos com alto risco de metástases linfonodais. Um desses tratamentos é a linfadenectomia durante a realização da gastrectomia, que tem sido objeto de controvérsia nas discussões acadêmicas nas últimas três décadas. Os cirurgiões japoneses realizam sistematicamente a linfadenectomia D2 por acreditarem que ela leva a um aumento na sobrevida, além de melhorar o estadiamento e, portanto, a uma melhor avaliação prognóstica. No Ocidente, o assunto ainda é controverso ${ }^{20}$.

$\mathrm{Na}$ Europa e países do Oriente, como Japão e Taiwan, encontramos uma prevalência maior de homens acometidos por câncer gástrico. Na nossa casuística, o número de homens e mulheres submetidos à cirurgia com linfadenectomia D2 foi similar.

Quando estudamos separadamente o sexo e a idade em nossa casuística não encontramos diferença estatisticamente significante nos dois grupos, entretanto houve uma média de idade cinco anos menor no sexo feminino (55,7 I anos nas mulheres e 60,79 anos nos homens).

A média de idade encontrada em nosso trabalho se aproxima à média de idade dos pacientes japoneses. Entretanto, note-se que, enquanto estamos operando cânceres avançados, os japoneses estão operando precoces. Se assim considerarmos, se estivéssemos operando tumores precoces, provavelmente estaríamos com pacientes com média de idade 5 ou 6 anos menor, já que este é o tempo estimado para um tumor precoce tornar-se avançado (T3 ou T4).
Quando estudamos a profundidade do tumor nos 57 pacientes operados, encontramos uma porcentagem semelhante de tumores $\mathrm{TI}$ e T2 (19,3\% e 17,5\%, respectivamente). A grande maioria (52,6\%) era de tumor T3 e apenas 10,5\% de tumor T4.

Nogushi et al. ${ }^{22}$, comparando resultados de instituiç̧ões japonesas e norte-americanas no que se refere à profundidade do tumor ( $T$ ), encontraram aproximadamente $50 \%$ dos pacientes japoneses classificados como TI contra apenas 20\% nos americanos. Encontraram também uma diferença importante quanto à localização do tumor, sendo quase $80 \%$ dos japoneses com tumor localizado no antro e corpo e apenas $20 \%$ localizados no terço superior do estômago. Já nos norte-americanos mais de 50\% dos pacientes apresentavam neoplasia localizada no terço superior do estômago.

Em nosso estudo, a grande maioria dos pacientes (43,9\%) apresentava linfonodos comprometidos tanto no nível $\mathrm{NI}$ quanto no nível N2. Em segundo lugar, tivemos pacientes com linfonodos livres de neoplasia tanto no nível NI quanto no nível N2 (35, I6\%), seguidos de pacientes com linfonodos comprometidos no nível $\mathrm{NI}$ e livres no nível N2 (19,3\%) e, finalmente, apenas um caso (I,8\%) que, apesar de apresentar os linfonodos $\mathrm{NI}$ livres de doença metastática, apresentava metástase no nível N2.

Esses dados revelaram que a grande maioria dos pacientes operados $(45,7 \%)$ se beneficiou da linfadenectomia D2, pois, caso tivesse sido realizada a linfadenectomia DI, todo este contingente ficaria com doenç, residual nos linfonodos do nível N2 que não teriam sido retirados. É interessante notar que em um caso houve metástase em linfonodos do nível N2 com linfonodos NI livres, demonstrando que a metástase pode não respeitar a seqüência natural, isto é, comprometer primeiro o nível NI. Neste caso, houve um "salto" da metástase diretamente para o nível N2. Isto nos levou a fazer algumas conjecturas: a) a metástase apenas seguiu um caminho diferente devido a uma particularidade da drenagem linfática daquele doente? b) será que, se utilizado - método da imunoistoquímica na detecção de metástases nos linfonodos, seria encontrada doença neoplásica também a nível NI?

Um fato é evidente: se realizada linfadenectomia DI neste paciente, este teria sido classificado como RO, isto é, livre de doença neoplásica e, em pouco tempo, haveria recidiva da neoplasia nos linfonodos.

Trinta e três pacientes ou $54,4 \%$ não apresentavam metástases em linfonodos no nível N2 e teoricamente estes não precisariam ter sido submetidos a linfadenectomia D2, porém $21 \%$ do total de pacientes apresentava câncer precoce do estômago ( $\mathrm{TI}$ ) e, nesta profundidade de tumor a metástase linfonodal é reduzida, tendo sido em nossa casuística igual a zero. Seguindo esta linha de raciocínio, sobrariam apenas $33,4 \%$ ou um terço dos pacientes que não se beneficiariam da linfadenectomia D2. Há que se pensar, no entanto, nos $45,7 \%$ de pacientes com N2 positivos que ficariam com doença residual no caso de não ser realizada a linfadenectomia D2.

Gotoda et al. ${ }^{21}$ estudaram 2570 casos operados por câncer precoce e mostraram um índice de aproximadamente 2,5\% de metástases linfonodais. Nesses casos de positividade em linfonodos, a grande maioria era em tumores maiores que $20 \mathrm{~mm}$, do tipo histológico indiferenciado e ulcerado. Nos tumores menores que $20 \mathrm{~mm}$, histologicamente diferenciados, a porcentagem de linfonodos comprometidos foi nula. 
Talvez tivéssemos encontrado metástases linfonodais nos nossos pacientes com tumor TI (precoce) se nossa casuística fosse maior. Como há controvérsia na literatura a esse respeito, é prudente que uma equipe treinada no tratamento do câncer gástrico realize linfadenectomia D2 mesmo no câncer precoce, principalmente quando este for maior que $2 \mathrm{~cm}$, estiver ulcerado ou invadir a submucosa.

Yoo et al. ${ }^{23}$ estudaram 2328 pacientes submetidos a ressecção curativa de câncer gástrico. Desse grupo, 508 pacientes tiveram recidiva tumoral precoce (antes de 24 meses) e foram estudados retrospectivamente quanto aos fatores de risco que levaram à recidiva. Os principais foram a invasão da serosa pelo tumor e a metástase linfonodal.

Shiraishi et al. ${ }^{24}$, analisando dois grupos de pacientes, um com recidiva precoce e outro com recidiva tardia e os fatores associados a essas recidivas, encontraram dois fatores estatisticamente significantes que influenciaram a recidiva precoce: o nível de linfonodos comprometidos (NI ou N2) e a extensão da linfadenectomia.

Refletindo sobre os trabalhos de Yoo et al..$^{23}$ e de Shiraishi et al. ${ }^{24}$, podemos atestar a importância da linfadenectomia estendida que, além de estadiar melhor, leva a uma melhor avaliação prognóstica principalmente quanto à recidiva.

O número de linfonodos extirpados por paciente em nosso estudo variou de I I a 103, com uma média de 28,63 linfonodos por indivíduo operado.

Nitti et al..$^{25}$ operaram 301 pacientes e encontraram um total de 799| linfonodos extirpados com variação entre 9 e 62 linfonodos, sendo a média de 27, 18 linfonodos por paciente.

Giovanetti et al. ${ }^{26}$ estudaram 267 pacientes gastrectomizados por neoplasia gástrica e mostraram que, quando a linfadenectomia realizada era a D0 ou DI, a média de linfonodos extirpada foi de 4, na linfadenectomia D2 foi de 36,5 e na D3 de 64,3 linfonodos.

Um dos objetivos do nosso trabalho foi estudar o estádio dos nossos doentes pela classificação da Associação Japonesa de Câncer Gástrico após a linfadenectomia D2 e, depois, simulamos hipoteticamente uma linfadenectomia DI nesses pacientes, isto é, consideramos apenas os linfonodos que teriam sido retirados se tivéssemos feito uma gastrectomia com linfadenectomia DI.

Foi possível observarmos fatos interessantes. Os tumores precoces $(\mathrm{TI}$ ), em número de II, não sofreram migração no estadiamento após a simulação DI. Os I I pacientes com estádio la com a linfadenectomia D2 continuariam la se tivéssemos realizado a linfadenectomia DI.

Quando analisamos os tumores T2 (dez pacientes) observamos que os classificados como lb (cinco pacientes) permaneceriam como lb após a simulação de linfadenectomia DI, assim como os classificados com estádio II (dois pacientes). Os classificados como Illa (três pacientes) migraram todos após a simulação DI, indo dois para estádio II e um para lb.

Nos tumores T3 (30 pacientes), quatro tinham estádio II e assim permaneceram após a simulação D I . Dezenove pacientes tinham estádio IIlb com linfadenectomia D2, sendo que I8 migraram para estádio Illa após a simulação. Sete pacientes que foram estadiados como Illa após a linfadenectomia D2 permaneceram inalterados após a simulação DI.
Nos tumores T4 (seis pacientes), dois eram estádio Illb e permaneceram inalterados após a simulaçãa e quatro migraram do estádio IV para o estádio Illb.

Notamos que só houve mudanças no estadiamento dos pacientes com câncer avançado do estômago (T2, T3 e T4), sendo que a grande maioria dos que sofreram migração foram os IIla, IIIb e IV. Percebe-se que esta migração sempre tendeu ao subestadiamento após a simulação de linfadenectomia DI.

Já nos cânceres avançados (T2+T3+T4), quando analisamos a classificação pela JCGA, notamos que os tumores lb e II permaneceram inalterados. Nos pacientes Illa, dos dez iniciais, três migraram para estádios menos avançados (30\%), sendo dois para estádio II e um para estádio lb. Nos pacientes inicialmente IIIb (2 I pacientes), houve uma grande migração ( 8 pacientes) para 0 estádio Illa. Nos quatro pacientes inicialmente classificados como estádio IV, todos migraram para o estádio IIIb.

Percebemos, portanto, que na nossa casuística a linfadenectomia D2 foi de grande importância para o correto estadiamento, principalmente nos cânceres avançados IIla, IIIb e IV. Nessas três categorias, dos 35 pacientes 25 migraram para estádios menos avançados após a simulação DI. Nesses pacientes, a sobrevida é maior quanto menor o estádio ${ }^{27}$.

Quando analisamos a concordância entre as duas classificações (D I e D2), observamos que as maiores discordâncias foram nos estádios Illa e Illb. Quando calculamos o p-valor comparando as duas classificcações (com linfadenectomia D2 e com simulação DI), encontramos p-valor geral $<0,00$ I, sendo, portanto, estatisticamente significante a diferença entre essas duas classificações.

Fuji et al.$^{28}$ analisaram os resultados de sobrevida em 5 anos em pacientes operados por câncer gástrico com linfadenectomia estendida classificados de acordo com a JGCA em 18 hospitais japoneses. Foram estudados mais de 26000 pacientes, sendo encontrada uma taxa média de sobrevida de $90 \%$ para estádio la, 85\% para Ib, 75\% para II, 50\% para IIla, 30\% para IIIb e 15\% para IV.

Se fizéssemos uma comparação desse estudo de Fuji et al. ${ }^{28}$ com o nosso, em que a grande maioria dos nossos pacientes de estádio IIIb, migraram para IIla e os de estádio IV na sua totalidade migraram para Illb, teríamos uma grande alteração na expectativa de sobrevida. Como os pacientes migraram para um estádio menos avançado, haveria uma expectativa de vida muito maior que se mostraria falsa com o tempo.

\section{Conclusão}

A linfadenectomia D2 é importante para o estadiamento mais apurado do câncer gástrico principalmente nos tumores avançados.

Os estádios que sofreram migração após a simulação D I foram IIla, IIIb e IV, sendo as mais freqüentes a IV e a IIIb. Nesses casos, houve tendência ao subestadiamento.

A linfadenectomia D2 evitou a presença de doença residual ( $N$ ) após a cirurgia em quase metade dos pacientes.

\section{Conflito de interesse: não há}




\section{SUMMARY}

\section{SiMULATION OF DI LYMPHADENECTOMY IN PATIENTS SUBMITTED to d2 lymphadenectomy. Prospective study of 57 patients WITH GASTRIC ADENOCARCINOMA}

BACKGROUND. To simulate a DI lymphadenectomy in 57 patients who had already been submitted to D2 lymphadenectomy, and analyze stage migration using the Japanese Gastric Cancer Association (JGCA) staging system.

METHODS. All patients underwent a D2 lymphadenectomy between August 1997 and November 200 I. At the end of surgery, the surgeon himselfseparated all lymph node stations. The parameters analyzed were: sex, age, lymph node invasion and number of dissected nodes. All patients were re-staged based on the new data, in order to evaluate the stage migration according to the JGCA staging system.

RESULTS. The advanced tumors corresponded to $80.70 \%$ and the early ones to $19.30 \%$. In $43.90 \%$ there were positive nodes at NI and N2 levels. The mean number of dissected lymph nodes was 28.63 in each patient. In IA, IB and II tumors, there were no changes in the stage after simulation of D I lymphadenectomy. In ten IIIA tumors, three migrated to stage II and IB; in 2 I tumors staged as IIIB, a migration was observed in I8; all stage IV tumors migrated to IIIB after the DI simulation.

CONCLUSIONS. a) D2 lymphadenectomy is important for the correct staging of gastric cancer; b) simulation of DI lymphadenectomy changed the stage of advanced tumors, particularly in stages IIIA, IIIB, and IN;c)D2 lymphadenectomy avoided residual lymph node disease in almost half of the patients operated. [Rev Assoc Med Bras 2006; 52(4): 270-4]

KEY WORDS: Lymph node excision adenocarcinoma. Stomach neoplasms. Gastrectomy.

\section{REFERÊNCIAS}

I. Lee JS, Douglas HO Jr. $\mathrm{D}_{2}$ dissection for gastric cancer. Surg Oncol 1997;6:215-25

2. Brasil. Ministério da Saúde. Instituto Nacional do Câncer. Estimativas da incidência e mortalidade por câncer no Brasil: São Paulo. [citado I 5 nov 2003]. Disponível em: http://www.inca.gov.br/estimativas/2003/ index.asp?link=tabela estados.asp\&UF $=S P$.

3. Volpe CM, Koo J, Moloro SM, Driscoll DL, Nava HR, Douglass H Jr. The effect of extended lymphadenectomy on survival in patients with gastric adenocarcinoma. J Am Coll Surg 1995; 181:56-64.

4. Maruyama K, Okabayashi K, Kinoshita T. Progress in gastric câncer surgery in Japan and its limits of radicality. World J Surg | 987; | |:4| 8-25.

5. Sasako M. Principles of surgical treatment for curable gastric cancer. J Clin Oncol 2003; $21: 274-5$

6. Iwanaga T, Furukawa H, Kosaki G. Relapse of early gastric cancer and its prevention. Rinsho Geka 1976;31:29-35. [abstract Medline]

7. Iwanaga $\mathrm{T}$, Koyama $\mathrm{H}$, Furukawa $\mathrm{H}$. Mechanisms of late recurrence after radical surgery for gastric carcinoma. Am J Surg | 978; | 35:637-40.

8. Lopasso FP. Câncer Gástrico: linfadenectomia, técnica, padronização e resultados. In: Gama-Rodrigues J.J, Lopasso FP, Del Grande JC, Safatle NF, Bresciani C, Malheiros CA, et al. editores. Câncer gástrico: aspectos atuais do diagnóstico e tratamento. São Paulo: Andrei; 2002. p. I89-2 I 2.

9. Msika S, Kianmanesh R. Treatment of gastric cancer. Chirurgie 1999; | 24:560-7.

10. Nakajima T, Yamaguchi T. Evidenced-based and data -based medicine (EBM and DBM) in practice guidelines for gastric cancer treatment. Lecture. In: Santoro E, Garofalo A. Gastric Cancer in the World 2003.
Proceedings of the 5th International Gastric Cancer Congress; 2003. Rome: International Gastric Cancer Association; 2003. p.95.

I I. Bunt AM, Hermans J, Van de Velde CJ, Sasako M, Hoefsloot FA. Fleure G, Bruijin JA. Lymph node retrieval in a randomized trial on western-type versus Japanese -type surgery in gastric cancer. J Clin Oncol 1996; | 4:2289-94.

12. Wu CW, Hsieh MC, Lo SS, Wang LS, Hsu WH, Lui WY, et al. Morbidity and mortality after radical gastrectomy for patients with carcinoma of the stomach. J Am Coll Surg 1995; | 81:26-32.

13. Sano T, Martin IG. Lymphadenectomy and pancreatico-splenectomy in gastric cancer surgery. Lancet I996;348(902 I): I 95-6.

14. Degiuli M, Sasako M, Calgaro M, Garino M, Rebecchi F, Mineccia M, et al. Morbidity and mortality after DI and D2 gastrectomy for cancer: interim analysis of the Italian Gastric Cancer Study Group randomized surgical trial. Eur J Surg Oncol 2004;30:303-8.

15. Yildirim E, Celen O, Berberoglu U. The Turkish experience with curative gastrectomies for gastric carcinoma: is D2 dissection worthwhile? J Am Coll Surg 200 I; 192:25-37.

16. Kodera Y, Yamamura Y, Shimizu Y, Torii A, Hirai T, Yasui K, et al. The number of metastatic lymph nodes: a promising prognostic determinant for gastric carcinoma in the latest edition of the TNM classification. J Am Coll Surg 1998; 187:597-603.

17. Ramacciato G, Aurelio P, D'angelo T, Cicchini C, Sternberg C.N. Does extended lymphadenectomy influence prognosis of gastric carcinoma after curative resection? Hepatogastroenterology 2000;47: 1 470-4.

18. Karpeh MS, Leon L, Klimstra D, Brennan MF. Lymph node staging in gastric cancer: is location more important than number? Ann Surg 2000;232:362-7I.

19. Palaj J, Konecny J, Petras L, Babiak L, Kukla K, Ciganak J, et al. Radical D surgery for patients with gastric cancer. Bratisl Lek Listy 2002; 103:424-7.

20. McCulloch P, Eidi Nita M, Kazi H, Tsuno Nagawa H, Gama-Rodrigues IJ. Gastrectomy with extended lymphadenectomy for the primary treatment of gastric cancer. In: Santoro E, Garofalo A, editores. Gastric cancer in the world 2003: proceedings of 5th International Gastric Cancer Congress. Rome; 2003. Rome: Edizioni Scientifiche Romane; 2003. p. I 01 -24.

21. Gotoda T, Yanagisawa A, Sasako M, Onoh, Nakanishi Y, Shimoda T, et al. Incidence of lymph node metastasis from early gastric cancer: estimation with a large number of cases at two large centers. Gastric Cancer 2000;3:219-25.

22. Noguchi Y, Yoshikawa T, Tsuburaya A, Motohashi H, Karpeh MS, Brennan MF. Is gastric carcinoma different between Japan and the United States? Cancer 2000;89:2237-46.

23. Yoo CH, Noh SH, Shin DW, Choi SH, Min JS. Recurrence following curative for gastric carcinoma. Br J Surg 2000;87:236-42.

24. Shiraishi N, Inomata M, Osawa N, Yasuda K, Adachi Y, Kitano S. Early and late recurrence after gastrectomy for gastric carcinoma: univariate and multivariate analyses. Cancer 2000;89:255-61.

25. Nitti D, Marchet A, Olivieri M, Ambrosi A, Mencarelli R, Farinati F, et al. Lymphadenectomy in patients with gastric cancer: a critical review. Tumori Suppl 2003;2:S35-8.

26. Giovanetti M, Brotto AC, Tiberio GA, Capedelli L, Vettoreetto N, Ronconi $\mathrm{M}$, et al. $\mathrm{D}_{2}$ lymphectomy in the treatment of gastric cancer: a retrospective view on our experience (1 990-1997). J Exp Clin Cancer Res 1999; | 8:455-8.

27. Kassab P, Castro OAP, Oliveira Neto RM, Ilias EJ, Issa M, Pirani WM, et al. Survival study of 74 patients submitted to $D_{2}$ gastrectomy. In: Santoro E, Garofalo A, editores. Gastric cancer in the world 2003: proceedings of 5th International Gastric Cancer Congress. Rome; 2003. Abstract. Rome: Edizioni Scientifiche Romane; 2003. p.72.

28. Fuji M, Sasaki J, Nakajima T. State of art in the treatment of gastric cancer: from the $7 \mathrm{I}^{\text {st }}$ Japanese Gastric Cancer Congress. Gastric Cancer 1999;2:151-7.

Artigo recebido: 28/04/05

Aceito para publicação: 02/03/06 